\section{THE SIGNIFICANCE OF THE FIXATION OF CERTAIN ABDOMINAL ORGANS IN THE HUMAN BODY *}

\author{
ROBERT C. COFFEY, M.D. \\ PORTLAND, ORE.
}

In studying the abdominal cavity from the standpoint of comparative anatomy we find that in man the capsule of the liver has fused with the diaphragm, while in the quadruped the liver is suspended by a mesentery the same as other organs. In man the duodenum is firmly fixed to the right abdominal wall, while in the quadruped it is freely movable. In man the ascending and descending colon and the two flexures are normally fixed to the posterior abdominal wall without the intervention of mesentery, while in quadrupeds the large intestine has a long mesentery and is therefore freely movable. In man the great omentum grows down over the transverse colon and adheres to it. This does not occur in the quadruped. In man the omental bursa is usually obliterated by adhesion of its layers together. Obliteration does not take place in the quadruped. In man the pancreas has been rotated behind the peritoneum and fixed to the abdominal wall. In quadrupeds the pancreas lies between the layers of the mesentery. In the monkey, which is a quadruped with a tendency to stand erect, the pancreas becomes adherent and the duodenum is more firmly fixed than in the original quadruped.

Going more into detail we find that in early fetal life, soon after the beginning of the formation of the alimentary canal, as it elongates, the duodenum, which at first has a mesentery or mesogastrium, turns over to the right carrying, with it the buds of the pancreas. The duodenum with its mesentery or mesogastrium lies down, so to speak, on the right posterior primitive parietal peritoneum and adheres to it. Thus the duodenum and pancreas become retroperitoneal and fixed. As the colon elongates the cecal end works its way upward and to the right and then down again, locating itself on the posterior and right lateral wall of the abdominal cavity, external to the kidney. The same position is assumed by the descending colon on the left side. At these points the ascending and descending colon and posterior layers of their mesenteries become adherent to the posterior parietal peritoneum, the endothelial surfaces of which become obliterated. Thus the ascending and descending colon not only fix themselves, but put in front of the kidneys the fibrous elements of two extra layers of peritoneum and the vascular and connective tissue structures of the mesenteries of these parts of the colon. The ascending and descending colon and their angles are not only fixed thus by adhesions, but are fixed on a shelf, having an angle of 51 degrees to the perpendicular. This leaves the transverse colon unsupported, except at the flexures and for its own mesentery, until the omentum grows down in front of it and attaches to it. This adds two more layers to its mesentery but does not give it any material support, owing to the fact that primarily the omentum is a sac and the transverse colon is only attached to the posterior layer. Later on, however, often not until nearly adult life, the omental bursa becomes obliterated up to the transverse colon, which gives the colon an extra support

* Read before the Section on Obstetrics, Gynecology and Abdomina Surgery at the Sixty-Sixth Annual Session of the American Medica Association, San Francisco, June, 1915. from the bottom of the stomach, in the form of a gastrocolic omentum. It is well to bear in mind that the gastrocolic omentum gives no support whatever to the colon until such peritoneal adhesion and consequent obliteration of the omental bursa takes place.

Unfortunately these adhesions do not always take place in man. Very rarely is it true that the duodenum and pancreas fail to adhere to the posterior abdominal wall. Occasionally the omental bursa completely fails to obliterate and permits the center of the transverse colon to swing from the flexure without the support of gastrocolic omentum, and occasionally this condition produces serious intestinal stasis. Occasionally the descending colon alone is not fused with the parietal peritoneum and produces, once in a great many cases, a unilateral left floating kidney. The relative infrequency of a left is probably due in a large measure to the costocolic ligament and the gastrosplenic omentum which hold the splenic flexure up much more firmly than the right is held. From 20 to 30 per cent. of human beings have an incomplete fusion of the cecum and ascending colon and its mesentery with the posterior parietal peritoneum. This deficient fusion is of several degrees.

First, the cecum and ascending colon alone may be mobile and very baggy and dilated, swinging from a hepatic flexure which is firmly fixed in its normal position. In a few of this type of cases a false membrane or veil has formed, known as Jackson's membrane. This type occurs so frequently and is so slightly mobile that it may be classed as normal.

In the second class of cases the colon has only partially migrated and has attached the hepatic flexure to the front surface of the kidney, leaving the cecum and ascending colon (while the patient is in the erect posture) swinging from the peritoneum on the front surface of the kidney. This peritoneal attachment has been described by Longyear as a distinct anatomic entity under the name of nephrocolic ligament. In some instances the weight of this baggy cecum dropping directly downward pulls the kidney out of position and produces the frequently seen right-side floating kidney. At other times the kidney holds its position, the pendulous colon swings from its attachment as a tube hung over a nail, producing an intermittent obstruction with consequent dilatation of the cecum and ascending colon, resulting in severe intestinal stasis, or the intestine may pull loose, leaving the kidney in its normal position while the hepatic flexure is mobile also. Some of these cases have become partially fixed by a Jackson's membrane.

In the third degree of nonmigration the hepatic flexure attaches to the front surface of the duodenum, when the pendulous baggy cecum with its fluid contents, especially in an individual who has become thin, at times pulls down so strongly on the duodenum as to kink the duodenum at the ligament of Treitz, and also at the junction of the first and second portion of the duodenum. Such a case shows a duodenal stasis with the Roentgen ray, the symptoms of gastric ulcer in the clinical history, and a dilated duodenum at the time of operation. We have seen such patients whose stomach symptoms were entirely relieved as soon as the cecum and ascending colon had been removed. We have seen other cases in which the same relief was obtained by fixation of the ascending colon and the first half of the transverse colon which makes the colectomy unnecessary. 
The fourth degree of nonfixation of the ascending colon is seen occasionally and is complete nonfusion in which neither the kidney nor duodenum has been covered by the mesocolon in which very extreme intestinal stasis existed which was relieved entirely by fixation operation by the method shown in the illustration.

In a certain per cent. of human beings there is a nonfixation of both the ascending and descending colon. These are the cases in which the so-called congenital type of enteroptosis occurs, in which both kidneys and all the hollow viscera tend to fall to the bottom of the abdomen, so that when the child reaches maturity the lower abdomen is dilated, the upper abdomen is contracted, the back is straight in an effort of the body to get in under this load and assume the carrying position. We contend that this typical picture of so-called congenital ptosis is due, in nearly all if not in all cases, to a deficient peritoneal $\mathrm{fu}^{-}$ sion, and that the peculiar body formation is nature's effort to make the best of a bad job. It is in these cases of nonfusion that all floating kidneys occur. We have been carefully watching for four years and have not found a single case of floating kidney which was not proved on the operating table to accompany a deficient fusion of the ascending or descending colon. In other words, a floating kidney is entirely a secondary condition, which accounts for the fact that the operation for floating kidneys has been considered a failure by most surgeons.

What is the remedy for these defects? In cases of deficient fusion on both the right and left sides, resulting in ptosis with the characteristic body formation, surgery is usually not advised nor indicated. Forced feeding, thereby increasing intra-abdominal pressure, with exercises such as Goldthwaite and also Martin have recommended, will produce the best results. Occasionally the symptoms of a patient suffering from general ptosis are so severe as to require surgical procedure, in which case nothing short of the following operation will do permanent good, namely, the fixation of the ascending and descending colon and the two kidneys, through two separate posterior incisions as described later.

Second, the opening of the abdomen and making a complete partition in the abdominal cavity by stitch- ing the omentum beyond the colon to the parietal peritoneum from one side to the other, thus making a water-tight partition, as we have had opportunity to see at a later operation.

After this operation has been completed the upper abdomen is expanded. Fortunately this very extensive operation is rarely necessary but if any surgery is attempted in the case of general ptosis with the body habitus described by Glenard and Goldthwaite, it is perfectly useless to do less. I have performed this complete operation for general ptosis six times with very satisfactory results, and with much improvement in the patient's health.

A right-side ptosis, however, calls for more frequent surgical interference. A right-side ptosis with a floating kidney accompanied by severe intestinal stasis, pain not relieved by dietary measures, admits of surgical treatment which consists first in making a posterior incision made just as if it were intended to remove the kidney and the first part of the ureter. Before the kidney is disturbed the peritoneum is opened, the appendix removed and the colon sutured to the parietal peritoneum. The peritoneal opening having been closed, the fat capsule of the kidney is opened, a door-flap hinge $11 / 2$ inches wide, of fibrous capsule of the kidney, is peeled off each side, a piece of the quadratus lumborum muscle as large as the middle finger is split off, two or three quilt chromic catgut sutures are passed through each flap of fibrous capsule, the two from the posterior flap are drawn around back of the separated strip of quadratus lumborum and tied to the corresponding sutures from the anterior, thus using the flaps of kidney capsule like a basket handle around the muscle. The posterior part of the fat capsule is now brought up through the same slit in the muscle and sutured to the front part of the capsule, thus making an additional basket handle of fat capsule.

There are also certain cases of severe intestinal stasis in which the kidney is not movable and in which the hepatic flexure may or may not be movable, giving severe pain in the right side or possibly dragging on the duodenum and producing symptoms of duodenal ulcer, but not relieved by any kind of dietary measures. Such a patient may be entirely relieved by suturing 
the cecum and ascending and first half of the transverse colon by its omentum to the parietal peritoneum. This, the most frequent operation indicated in the dealing with all defects of peritoneal fusion, is performed as follows: A long right rectus incision is made to expose the entire length of the cecum and ascending colon. If any false bands or membranes have formed they should be cut and a series of pursestring sutures of very fine linen or silk passed but not tied, beginning at the cecum. Each purse-string suture should begin at the true parietal peritoneum to true peritoneum of the colon preferably including a small bight of a longitudinal band. Small bights of the intervening loose tissue should be included. These sutures should be placed every $1 / 2$ to $3 / 4$ inch back toward the normal location of the kidney where the hepatic flexure is fixed. Then the intestine is turned forward and attached by its loose peritoneal connective tissue to the lateral peritoneum to within an inch of the incision and also near to the beginning of the omentum. At this stage of the operation the wail on the median side of the incision is lifted and the omentum sutured to the anterior parietal peritoneum from the median line backward to meet the other line of sutures. The omental sutures are of chromic catgut to avoid the danger of extension of a possible infection of the abdominal wound to the line of linen sutures which in two of my early cases kept up a discharging sinus for months until the linen sutures came out.

Occasionally a very severe case of constipation is due to the lack of obliteration of omental bursa, and cannot be cured by either dietary or medical means, but is entirely cured after suturing the omentum to the abdominal wall, thereby at once obliterating the omental bursa and holding up the middle of the transverse colon, which prevents the drag of a loaded transverse colon on the flexures.

\section{CONCLUSIONS}

1. Prenatal fixation of the pancreas, duodenum, ascending and descending colon and their mesenteries to the parietal peritoneum; of the liver to the diaphragm; of the omentum to the transverse colon; and the obliteration of the omental bursa, are evolitionary changes which help to fit man for the erect posture are not found in quadrupeds.

2. All cases of floating kidney with general visceroptosis are found in patients whose ascending and descending colon with mesenteries have failed to properly adhere to the parietal peritoneum. Surgical fixation of a floating kidney without fixing the colon is not based on sound surgical and anatomic principles; hence the failure of such operations in the past and necessity for surgical fixation of the colon at the same time.

3. Many cases of intestinal stasis are caused by the disturbed relations of the parts of the intestinal tract resulting from normal fixation of the ascending colon. While most of such cases are relieved by medical means, a few require surgical fixation.

Surgical fixation of the ascending colon should be extended to the suspension of first portion of the transverse colon by the omentum, for purpose of preventing harmful angulation at the hepatic flexure.

4. Some cases of persistent stomach trouble are due to the weight of a distended mobile cecum dragging on the third portion of the duodenum, and are relieved by anchoring the ascending colon and suspending the first half of the transverse colon.

789 Glisan Street.

\section{ABSTRACT OF DISCUSSION}

Dr. Max Einhorn, New York: I am glad that Dr. Coffey does not commend these operations for enteroptosis. From my experience in enteroptosis, which extends probably over a period of twenty-seven years, I would say that I have not yet seen cases in which an operation was distinctly imperative. Enteroptosis, as such, does not demand surgical intervention. Enteroptosis itself is not of such great consequence nor does it interfere in such degree with the well being of the patients, and we can always do something to relieve them of their symptoms.

It is impossible to fasten each organ in the abdomen. I think we should take it for granted that, having developed from quadrupeds and assumed a standing position, nature probably in the course of the development has already accomplished what we require, and we do not need to worry about it at all or try to imitate those quadrupeds. I do not think they are any better off than we, so that if some organs are not entirely fixed in the abdomen that does not show that they are diseased or that we should change them. A flexible organ can sometimes do its function very well, and if we try to change it we sometimes do harm. That we should bear in mind when we want to interfere.

As to the medical treatment of enteroptosis there are symptoms sometimes requiring correction, and one should try to build up the patient. A great many of these patients with enteroptosis suffer from bad nutrition. They are afraid to eat, and owing to the lack of fat they have those symptoms. If we make them eat and support the abdomen (sometimes such a support is not urgent and they can do without it) we pull the organs up and they improve and we bring about a change in the position of those organs, especially where the enteroptosis has developed after some sickness; the loosening up of the abdominal muscles through this added fat will do wonders. If we build them up, with fat and muscles the organs very often go back of themselves to their natural positions. So in these simple means of nourishing a patient we have our ideal remedy at hand to cure a great many of these patients without surgical intervention.

Dr. Albert Goldspohn, Chicago: While the previous speaker was speaking there occurred to me what has frequently been a paradox to me in the feeding question, namely, if these patients do not eat there is something wrong about their gastro-intestinal apparatus causing lack of appetite or fear of the distress which they would afterward realize. Now, if they are to be permitted to walk about on two feet and not be compelled to go on four, how is the physician to succeed in getting them to eat? And if they are crowded to eat more than they want, is there a proportionate increase in digestion and assimilation following forced feeding?

We all operate for floating kidney rather infrequently. There is probably no operation that I do less frequently than that, and as Dr. Coffey has said, simply fixing the kidney and ignoring the colon, especially when this is done by various uses of the fibrous capsule alone or chiefly, is extremely uncertain in its duration and very unsatisfactory in its results. Dr. Longyear, in suggesting the connection between the kidney and the colon, and speaking of his nephrocolonic ligament, made a step which leads us to think of a possible cause for this dissatisfaction, but I, like many other men, have not been able to trace that ligament. I know, however, that there is a united descent between the kidney and the ascending colon; that the colon is dislocated with the kidney; and I knew that before I had learned of Dr. Coffey's explanation. The technic which I have resorted to, therefore, is chiefly that suggested by Dr. Edward Andrews of Chicago, which consists in making a hammock back of and beneath the kidney out of the fatty capsule and making use of the fibrous capsule only as an adjunct or incidentally. By drawing forward and upward the fatty capsule gathered together on both sides of the kidney, we raise the colon likewise if there is a connection between the two, and by fastening the capsule 
like the meat in a sandwich, between the muscles in closing the wound by mattress sutures, we can make a fixed and permanent anchorage. This process in the operations that I have performed for floating kidney has been very satisfactory; and $I$ think it coincides with the reasoning and the technic which Dr. Coffey suggests. I think it will be even better to do as he does: enter the abdomen from behind and laterally and anchor the colon and then do this work as he does it on the kidney.

Dr. A. N. Creadick, Portland, Ore.: Dr. Coffey's paper discussed one type of ptosis, namely, congenital, and not the acquired type such as we see in postpuerperal cases, with lost tone of the ventral abdominal wall, a flaccid lower abdomen and the resultant symptoms caused thereby. Let us lay down two or three simple rules for ptosis that will meet with common approval. First, ptosis is established when any supra-umbilical viscus is permanently and wholly below the level of the umbilicus, is then provocative of the usual course of symptoms and requires surgical intervention. No remedy can be applied to this type except the prostrate position or surgical procedure-some such operation as this devised by Dr. Coffey. Second, the "acquired ptosis" patients have some hope in medical treatment of their general asthenic state. The result of such medical treatment is very satisfactory in our hands. A large number of patients are relieved by medical treatment alone. Third, the factors which cause ptosis of the acquired type consist of (a) posture, the loss of the supporting shelf of the spinal column, which Dr. Coffey describes as an angle of $55 \mathrm{de}$ grees from the horizontal; (b) the tone of the musculature of the ventral abdominal wall and (c) subperitoneal fat. In acquired ptosis the posture can be corrected by gymnastics, which also tone up the abdominal wall as well. ( $I$ use the frame devised by Dr. Martin of Chicago, elevating the foot to a window sill, securing an exaggerated Trendelenburg position, then requiring the patient to do regular leg exercises.) Hot compresses on the abdomen and forced feeding are useful.

DR. H. O. Pantzer, Indianapolis: I proceed on a theory just the opposite of Dr. Coffey's. Instead of further fixing these organs for cure, I loosen all such angulations, kinks and distortions as interfere with function. In other words, in effect, I choose rather to reestablish the old quadruped condition. I may say that the clinical results are at least equally as satisfactory as those obtained by the fixation methods, which, too, in single cases, are not all we could wish for.

Dr. R. C. Coffey, Portland, Ore.: I never think of operating in these cases until the abdomen is black with blisters which the internist has produced with hot packs during forced-feeding process. The first thing is to have the internist try them out. My colleague Dr. Jones has an institution devoted to the forced feeding treatment. Occasionally there is a case that will not be relieved for mechanical reasons. I know an internist in my city who claims, like Dr. Einhorn, that he can cure them all, but I will say that many of the cases I get come through his hands.

A great many of our best surgeons are inclined to call everything general ptosis. Now Dr. Creadick spoke of acquired ptosis. There is the most definite ptosis seen in the abdomen. The ascending and descending colon have blended in perfectly with the parietal peritoneum and remain in normal position, but the stomach and the middle of the transverse colon have dropped down. This is the most troublesome type of ptosis. It has nothing to do with the subject of my paper and the majority are cured with fattening. Fattening will cure nearly all these cases by increasing the intra-abdominal pressure. The type we are discussing in the paper is an embryologic defect.

\section{ROENTGEN OBSERVATION OF THE GALLBLADDER AND HEPATIC DUCTS AFTER PERFORA- TION INTO THE DUODENUM}

\section{R. D. Carman, M.D., Rochester, Minn.}

The roentgenologic demonstration of a barium-filled ga!1bladder in a living patient is sufficiently unusual to warrant its report. So far as I am aware, no similar case confirmed by operation or necropsy has appeared in the Roentgen literature.

The patient, a woman aged 54 , had had a cholecystostomy for gallstones in January of this year, elsewhere. She came to the Mayo Clinic May 22. In the scar of the operation wound were two sinuses, discharging pus, and surrounded by a movable, nodular mass. The total acidity was 4 , all combined; no food remnants. Hemoglobin 80 per cent. The clinical s y m tom s consisted chiefly of some hunger distress, belching and watery regurgitation.

At the Roentgen examination no retention from the six-hour meal was found. When the stomach was filled with the second meal, the pyloric end showed a smooth, concentric filling defect, giving the lumen a conical form, and typical of scirrhous carcinoma. Far up in the right abdominal quadrant was a dense collection of barium with sprig-like branches (Fig. 1). From its situation this was believed to be in the gallbladder, indicating a
communication between it and the upper intestinal tract, which was reported to the clinicians in charge of the casc. The tendril-like branches were deemed to be due to the extension of barium into the hepatic ducts.

At operation (E. H. Beckman, June 28, 1915) the external sinuses were found to lead into a dense carcinomatous mass surrounding the gallbladder and involving the stomach. By reason of its extent the cancer was not exsectable. The gallbladder itself, which was excised, simply showed a chronic cholecystitis with particles of barium adherent to the mucosa. The fundus of the gallbladder communicated with the duodenum through a perforation.

At necropsy on the following day the perforation, which had been covered by the surgeon as well as possible, was found in the anterior wall of the first portion of the duodenum, toward the former site of the gallbladder. Metastases were noted in the liver. Some barium was seen in the hepatic ducts.

Cooperation.-Cooperating with an efficient health officer, physicians can make the efforts of the health department successful or their success impossible.-Trask. 